\begin{tabular}{lllllllllllllllllllllllllllllllll}
\hline$R$ & $E$ & $V$ & I & S & T & A & D & E & E & S & T & U & D & I & O & S & I & N & T & E & $R$ & N & A & C & I & O & N & $A$ & A & $E$ & $S$
\end{tabular}

\title{
Los empresarios chilenos frente a la región Asia-Pacífico*
}

\author{
Manfred Wilhelmy von $W^{* *}$
}

\begin{abstract}
Si bien Chile es un actor pequeño en el comercio internacional (el total de su intercambio no pasa del 0,28\% del comercio mundial), la economía chilena se ha abierto considerablemente, tanto en los aspectos comerciales como financieros, muestra un interesante desarrollo del sector exportador y una receptividad a productos y servicios de origen externo, y ha consolidado un régimen no discriminatorio para las inversiones extranjeras, todo lo cual ha contribuido significativamente a su trayectoria de crecimiento.

En los últimos quince años, los empresarios chilenos, protagonistas de estos procesos, han desarrollado vinculaciones económicas más allá de los escenarios habituales constituidos por los mercados de América Latina, abriéndose a regiones como Asia-Pacifico.
\end{abstract}

\section{Planteamiento General}

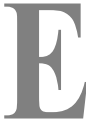

1 objetivo de este artículo es lograr un diagnóstico y ofrecer algunas sugerencias sobre el rol actual y potencial de las empresas de nuestro país en la región Asia-Pacífico. Nuestro análisis no enfatizará el papel empresarial en la política exterior económica, sino que procurará describir y explicar los factores por los cuales esta región adquiere gran importancia para el sector privado, y puede en el futuro tener una gravitación aún mayor.

Primero, es necesario hacer una precisión en los términos. En un sentido amplio, Asia-Pacífico es el conjunto de 21 economías de Asia, Oceanía, América del Norte y del Sur que forman parte del foro APEC (Asia Pacific Economic Coopera-

* Los juicios expresados por el autor son personales y no representan puntos de vista institucionales de la Fundación.

${ }^{* *}$ El autor agradece especialmente la valiosa colaboración de Hernán Gutiérrez y la acuciosa revisión final del texto por Fernanda Hurtado. 
tion), fundado en 1989, al que Chile ingresó entre 1993 y 1994, y que preside durante el año 2004.

Sin embargo, en estas páginas, AsiaPacífico tiene una acepción menos amplia, aunque todavía muy considerable: comprende el conjunto de economías situadas en la ribera opuesta del Pacífico, desde Japón y Corea en el norte, pasando por el mundo chino (República Popular China, Hong Kong y Taiwan) y el sudeste asiático (Indonesia, Malasia, Singapur, Tailandia, Filipinas y los países de Indochina), hasta Australia y Nueva Zelandia en el sur.

\section{Los interlocutores chilenos} permiten que los grandes conglomerados de Japón desarrollen negocios con buenas perspectivas inmediatas y de largo plazo.

La idea central que me propongo desarrollar es que en las relaciones de la empresa chilena con la región se da una paradoja. Por una parte, ya existe una importante tradición de vinculaciones empresariales de Chile con la región. Por ejemplo, en el caso de Japón, la mayor economía de Asia y la segunda en tamaño en el mundo, las empresas de nuestro país tienen una relación con el sector privado de ese país que puede calificarse de privilegiada. Grandes conglomerados de Japón valoran en alto grado los vínculos empresariales con sus contrapartes chilenas, ya que consideran que sus interlocutores nacionales son actores confiables, con los cuales es posible y conveniente (rentable) desarrollar negocios en una perspectiva no sólo de ganancia inmediata, sino también de largo plazo.

Pero, al mismo tiempo y desde la perspectiva de toda la región, se trata de un conjunto de relaciones que todavía se encuentra en una fase primaria de desarrollo. Esto ocurre por varias razones, a saber:

1. En comparación con otras áreas geográficas y económicas, la orientación al Asia-Pacífico sigue siendo patrimonio de un número relativamente reducido de empresas. Por ejemplo, en el área NAFTA operan aproximadamente cinco mil exportadores, mientras en el Asia-Pacífico son unos mil (en ambas regiones hay una parte que es estable y otra que va rotando, principalmente pymes, que son exportadoras ocasionales).

Por lo general, una lista muy corta de empresas (tal vez 10 o 12) representan más del $90 \%$ de las exportaciones a cada uno de los mercados de destino en AsiaPacífico. Las demás, por lo tanto, se reparten un porcentaje muy pequeño de cada mercado. Para estos últimos exportadores, los costos de transacción inciden fuertemente en la rentabilidad del negocio. Si esos costos son elevados, como ocurre cuando la distancia es grande y el medio comercial poco conocido, es probable que la exportación no deje utilidades interesantes. En consecuencia, la disminución de los costos de transacción comercial con la región Asia-Pacífico debe ser un tema prioritario para estimular a más empresas a entrar a los mercados asiáticos. 
2. La "canasta" de productos exportados al Asia-Pacífico todavía se concentra más en los rubros de commodities y menos en los productos industriales que en otras regiones de destino de los productos chilenos. Pese a que se van incorporando nuevos bienes, la diferencia todavía es clara: en el año 2002, las exportaciones de manufacturas al Asia-Pacífico bordearon los cien millones de dólares, mientras en el área andina fueron del orden de los 700 millones ${ }^{1}$.

\section{La disminución de los costos de transacción debe ser tema prioritario para las empresas que deseen entrar al mercado asiático.}

3. Entre los factores que explican esta situación, algunos son objetivos. De los primeros, mencionemos la distancia geográfica, la escasez de inteligencia de mercado efectivamente utilizada por las empresas, y las estructuras arancelarias que perjudican el potencial de las manufacturas en los países con los cuales Chile no tiene acuerdos comerciales preferenciales (por eso es tan importante que el país logre más acuerdos de este tipo en AsiaPacífico, como lo ha hecho en el resto del mundo).

A los anteriores hay que agregar problemas coyunturales como la crisis asiática del período 1997-1999, que hizo pensar a algunos, por supuesto erróneamen- te, que las economías de Asia-Pacífico se estancarían a largo plazo, y el tema del SARS, flagelo que en el año 2003 perjudicó seriamente a economías como Hong Kong y China.

Otros factores son de carácter subjetivo, principalmente la percepción de una mayoría de empresarios de falta de familiaridad y de preparación para incursionar en medios económicos y comerciales que les resultan desconocidos, y aun en cierto grado "misteriosos". Este problema es consecuencia de la marcada brecha cultural que persiste entre nuestro país y el Asia-Pacífico, que se refleja en el mundo de los negocios y que sólo puede solucionarse con un trabajo a largo plazo, que debe incluir tanto buenos seminarios sobre cómo hacer negocios con determinados países y el conocimiento directo de estos países mediante viajes adecuadamente programados y organizados. Debo subrayar especialmente este punto, ya que en el mundo asiático el conocimiento personal se considera esencial para establecer la confianza indispensable para contraer y cumplir compromisos comerciales y financieros.

En esta materia, salvo notables excepciones, nuestros empresarios todavía tienen una visión insuficientemente desarrollada acerca de la región Asia-Pacífico y que va a la zaga de la importancia real que ha alcanzado esta región del mundo. Uno de los objetivos implícitos de la presidencia chilena del APEC es contribuir a cambiar esta impresión. Se

${ }^{1}$ DIRECON, Ministerio de Relaciones Exteriores, Comercio Exterior de Chile, tercer trimestre 2003, noviembre 2003; pp.31 y 55. 
trata de que las empresas de nuestro país logren posicionarse mejor en el Asia-Pacífico, lo que debería redundar en más y mejores negocios y, por tanto, en mayores utilidades y en la creación de empleos de buena calidad.

\section{Los mercados asiáticos se encuentran en fuerte expansión debido a las elevadas tasas de crecimiento de sus economías.}

\section{Asia Pacífico como conjunto de MERCADOS DE EXPORTACIÓN}

Una primera mirada empresarial al Asia-Pacífico considera a esta región como un gran conjunto de mercados de exportación, que ofrece un campo fértil para los productos chilenos. Esto ya es una realidad, porque Chile destina cerca de 30\% de sus exportaciones al área.

Sin duda, esta cifra ya es un logro importante. A modo de comparación, observamos que Brasil destina sólo un 17\% de sus exportaciones al Asia-Pacífico (aunque las cifras absolutas sean mayores) y que, como señala en un reciente informe la Fundación Okita de Argentina, las exportaciones de este último país a Japón representan apenas una séptima parte de las chilenas ${ }^{2}$.

Pero tal vez más importante que lo anterior es el hecho de que estos mercados se encuentran en fuerte expansión, debido a las elevadas tasas de crecimiento de las economías respectivas y al proceso de apertura de éstas al comercio exterior, que es más rápido que la apertura en otras partes del mundo. Parte de este proceso se debe sin duda al APEC, el foro de la región en que nuestra presencia ha resultado muy beneficiosa para el país y que puede reportarnos ventajas adicionales a corto plazo, si Chile aprovecha el año de su presidencia del APEC.

El claro interés nacional por intensificar las relaciones de las empresas chilenas con la región Asia-Pacífico se sintetiza en los conceptos de tamaño, dinamismo, y complementariedad.

En cuanto al tamaño, nos referimos a más de un tercio de la población mundial, a una cuarta parte del PIB mundial, y a un tercio del comercio exterior global. El dinamismo se refleja en tasas de crecimiento superiores al 7\% real anual desde 1990 (exceptuando a Japón), y a un crecimiento proyectado de un $6 \%$ en lo que resta de esta década, con tasas de expansión comercial superiores a las del crecimiento del producto. Este ritmo de expansión implica un fuerte incremento de las poblaciones de los segmentos de clase media y media-alta, de gran poder adquisitivo, deseosos de acceder a productos importados. Estos segmentos crecen más rápidamente que el total de los habitantes de cada país, configurando nuevos mercados de la mayor importancia.

La complementación actual y potencial de las economías es evidente. Países como Japón, Corea, China, Singapur y

\footnotetext{
${ }^{2}$ Fundación Okita, Asia: la Gran Oportunidad, Buenos Aires, abril 2003; p. 2.
} 
otros, son grandes demandantes de recursos naturales con diferentes grados de elaboración, en los cuales la economía chilena tiene importantes ventajas y es proveedora de manufacturas altamente competitivas con fuentes alternativas tradicionales -no asiáticas- de abastecimiento.

\section{En Asia-Pacífico aún hay mercados emergentes que recién se abren a las exportaciones.}

Además, mientras la economía chilena es deficitaria en su nivel de ahorro interno, en Asia-Pacífico la situación es exactamente opuesta, por lo que en esta región se generan importantes recursos disponibles para la inversión extranjera. Para aprovechar parte de estos recursos, es necesario aprender a interesar a los inversionistas potenciales, tarea que en nuestro país se encuentra en gran medida pendiente. Entre estas inversiones y el comercio exterior se pueden producir interesantes sinergias. Así ocurre, por ejemplo, cuando inversionistas extranjeros invierten con el fin de potenciar empresas locales que destinan parte importante de sus productos a la exportación. Pero, a la inversa, también es necesario considerar seriamente proyectos de inversión chilenos en la región Asia-Pacífico. Por ejemplo, inversiones en centros de distribución estratégicamente situados y eficientemente gestionados pueden ayudar a disminuir las desventajas de Chile frente a algunos competidores que se encuentran más cerca de los mercados finales.

La posición de Chile frente a Asia-
Pacífico difiere de las relaciones comerciales con mercados maduros, como los de la Unión Europea y Estados Unidos. Por supuesto, estos ofrecen interesantes oportunidades a nuestros productos y servicios, y brindarán otras nuevas en el contexto de los respectivos acuerdos de libre comercio. Pero se trata de mercados que ya han sido más penetrados y que están saturados de importaciones en algunos rubros, $\mathrm{y}$, por otra parte, la liberalización competitiva (o sea, la celebración de un número cada vez mayor de tratados de libre comercio) tenderá a erosionar en el tiempo las ventajas que Chile ha logrado mediante los acuerdos. En cambio, en Asia-Pacífico todavía es posible encontrar una serie de mercados emergentes, sumamente dinámicos, que recién se están abriendo masivamente a las importaciones y que, por lo mismo, resultan particularmente atractivos.

\section{EXPORTACIONES CHILENAS POR REGIONES DE DESTINO}

En el año 2002, la distribución regional de las exportaciones chilenas fue la siguiente:

\begin{tabular}{|l|c|c|}
\hline $\begin{array}{c}\text { REGIÓN DE } \\
\text { DESTINO }\end{array}$ & $\begin{array}{c}\text { VALOR } \\
\text { EXPORTADO }\end{array}$ & $\begin{array}{c}\% \text { DE } \\
\text { EXPORT. DE } \\
\text { CHILE }\end{array}$ \\
\hline Asia Pacífico & US\$ 4.746 & $26,9 \%$ \\
\hline NAFTA & US\$ 4.840 & $27,4 \%$ \\
\hline Unión Europea & US\$ 4.286 & $24,2 \%$ \\
\hline Mercosur & US\$ 984 & $5,6 \%$ \\
\hline Comunidad Andina & US\$ 1.339 & $7,6 \%$ \\
\hline
\end{tabular}

Datos del Banco Central de Chile, en millones de dólares de los Estados Unidos 
Se observa una distribución bastante pareja entre las tres principales regiones de destino de las exportaciones, con una leve diferencia a favor del NAFTA respecto de Asia-Pacífico.

En el primer semestre de 2003, esta situación cambió, ya que Asia-Pacífico se sitúa en un claro primer lugar con 2.987 millones de dólares $(29,6 \%)$, aventajando al área NAFTA, que registra 2.549 millones $(25,3 \%)$ y a la Unión Europea con 2.502 millones de dólares $(24,8 \%)$. Esto sugiere que Asia-Pacífico ha recuperado el primer lugar que tuvo en forma clara hasta la crisis asiática que estalló en 1997, cuando las exportaciones a dicha región alcanzaron el récord histórico de 5.711 millones de dólares $(33,6 \%)^{3}$.

\section{Pese a los últimos progresos,} seguimos siendo pequeños como exportadores a los grandes mercados del Asia-Pacífico.

\section{PARTICIPACIÓN EN LOS MERCADOS DE DESTINO}

Sin embargo, las cifras recién citadas no son suficientes. Es interesante realizar un cálculo de los porcentajes que representan las exportaciones chilenas en el total de las importaciones de algunos países del Asia-Pacífico. Este análisis muestra que la participación de los productos chilenos en los mercados de destino todavía es muy baja, especialmente en los países del sudeste asiático y en Australia y Nueva Zelandia.

2002: EXPORTACIONES CHILENAS EN MILLONES DE DÓLARES Y COMO \% DE LAS IMPORTACIONES DE CADA PAÍS

\begin{tabular}{|l|lr|c|}
\hline Japón & US\$ 1.947 & $(0,48 \%)$ \\
\hline $\begin{array}{l}\text { República } \\
\text { Popular China }\end{array}$ & US\$ 1.240 & $(0,38 \%)$ \\
\hline Corea & US\$ 711 & $(0,39 \%)$ \\
\hline Taiwan & US\$ 531 & $(0,47 \%)$ \\
\hline Indonesia & US\$ 64 & $(0,12 \%)$ \\
\hline Tailandia & US\$ 50 & $(0,07 \%)$ \\
\hline Australia & US\$ 60 & $(0,07 \%)$ \\
\hline
\end{tabular}

Cabe advertir que en algunos mercados la participación puede ser un poco mejor que los porcentajes presentados, porque algunas cifras de importaciones en los destinos incluyen no sólo bienes, sino también servicios. Pero esta prevención metodológica no altera sustancialmente el cuadro, que nos indica que, a pesar de los grandes progresos logrados en los últimos 10 a 15 años, seguimos siendo muy pequeños como exportadores a los grandes mercados de Asia-Pacífico. Naturalmente, tanto en lo concerniente al aprovechamiento de nuevas oportunidades como a los obstáculos al acceso a los mercados y a la competencia de terceros, hay múltiples situaciones específicas que no podemos considerar en estas páginas ${ }^{4}$.

En todo caso, el punto central es que para una economía exportadora pequeña como la chilena, bajos índices de penetración significan que hay espacio para crecer sin modificar radicalmente las situaciones de los mercados comer-

\footnotetext{
${ }^{3}$ Fuente: DIRECON, Departamento de Estudios.

${ }^{4}$ Fuente: Fundación Chilena del Pacífico, Perfiles Económicos Asia Pacífico 2003, Anexo 14.
} 
ciales que interesa desarrollar, lo que limita el riesgo de reacciones muy fuertes de los competidores (nuevamente, esta es una afirmación general: la situación puede ser distinta tratándose de productos donde Chile tiene mayor importancia). Por ejemplo, sería una interesante meta de mediano plazo llegar al $1 \%$ de participación en las importaciones de los principales mercados de Asia- Pacífico. Ello implicaría más que duplicar nuestra presencia actual en Japón, China, Taiwán y Corea, y multiplicarla por un factor de ocho o más en otros mercados. Sería, sin duda, una meta muy ambicios a .

A continuación, algunas observaciones sobre mercados específicos.

\section{JAPÓN, SEGUNDO DESTINO DE LAS EXPORTACIONES CHILENAS}

Japón sigue siendo el segundo destino global de las exportaciones chilenas. Como es sabido, la economía japonesa ha permanecido estancada durante una década, aunque en el año 2003 se dio un cambio positivo, fruto de iniciativas de reforma del gobierno del primer ministro Koizumi y de mejores perspectivas internacionales. Las empresas chilenas deben estar atentas a estas señales, porque una recuperación podría reactivar la demanda por nuestros productos. Es necesario recordar que en 1995 las ventas a Japón bordearon los 3 mil millones de dólares, o sea, en un contexto de reactivación se abre un gran espacio de crecimiento.
La economía japonesa está saliendo de su estancamiento gracias a las reformas del gobierno y a la mejora de las perspectivas internacionales.

Para mejorar el acceso al mercado, los empresarios nacionales cuentan con instrumentos como el Comité Empresarial Chile-Japón, que facilita valiosos contactos y analiza escenarios que capacitan a los empresarios para entender ese exigente y competitivo mercado. Aunque lo más conveniente sería que Chile tuviera acceso preferencial en virtud de un tratado de libre comercio, este tema está postergado, pero no descartado. En su sesión celebrada en Santiago en noviembre de 2003, los miembros japoneses y chilenos del Comité acordaron por unanimidad retomar la iniciativa de celebrar un TLC. Este acuerdo se ha comunicado al presidente Lagos y al primer ministro Koizumi. Entretanto, deberemos perseverar en la competencia exportadora sin preferencias bilaterales. Esto es muy importante, aun más allá de Japón, ya que cuando Japón se abre a un determinado producto -por ejemplo, si determinada fruta fresca es declarada apta para el consumidor japonés- las autoridades de los demás mercados de la región consideran que la señal japonesa es un antecedente para tomar sus propias decisiones sobre el producto.

En la medida en que Japón sigue liderando la región Asia-Pacífico, sus compañías de comercio exterior (las sogoshosha, como Itochu y otras) pueden 
servir a los exportadores chilenos no sólo para llegar al mercado japonés, sino también para penetrar el resto de la región una vez que las empresas consideren que se trata de proveedores confiables, capaces de entregar productos que cumplan rigurosamente con las especificaciones para cada mercado, y que tengan precios competitivos.

\section{Para incrementar los lazos entre América Latina y el este de Asia hay que aprovechar mejor las redes de distribución de las empresas japonesas.}

En un estudio reciente efectuado para el gobierno de Singapur por la prestigiosa consultora Sreekumar \& Siddique, una de las recomendaciones principales para incrementar los lazos comerciales entre América Latina y el este de Asia es aprovechar mejor las grandes redes de distribución de estas empresas niponas tradicionales ${ }^{5}$.

A modo de conjetura podría agregarse, por último, que el hecho de que próximamente entrará en vigor el TLC con Corea podría hacer que los empresarios japoneses temieran un desplazamiento del mercado chileno hacia sus competidores coreanos, y por otros que gozarán pronto de preferencias, lo que podría provocar una movilización política ante las autori- dades de Tokio en favor de un TLC entre ambos países.

\section{RÁPIDO ASCENSO DE CHINA COMO MERCADO}

El deterioro de las exportaciones a raíz de la crisis asiática se compensó en gran medida gracias al dinámico comportamiento de este mercado, que aumentó de 133 millones de dólares en 1994 a 1.240 millones en 2002 (9,3 veces), y llegó a constituir el segundo mercado en Asia-Pacífico y el tercero a nivel global. Este extraordinario crecimiento se debe al fuerte desarrollo de la economía china, que se tradujo en una gran expansión de la demanda china de insumos para la industria manufacturera, como cobre y otros productos, pero más recientemente también en mayores compras de bienes de consumo para la nueva clase media urbana y el mercado de ejecutivos internacionales y turistas ${ }^{6}$.

La relocalización de industrias taiwanesas en el continente, que tuvo por objeto aprovechar el bajo costo de la mano de obra, también ha contribuido a incrementar la demanda china, a la vez que ha disminuido el dinamismo de las importaciones de Taiwán.

La incorporación de China a la OMC es muy importante para Chile debido a que somete al país a las disciplinas co-

\footnotetext{
${ }^{5}$ Sreekumar \& Siddique, FEALAC Transpacific Trade and Investment: Overcoming Obstacles and Impediments -An Independent Study Submitted to the Ministry of Foreign Affairs, Singapur, octubre 2002, p. 29.
}

${ }^{6}$ Fundación Chilena del Pacífico, Perfiles 2003. 
merciales de esta entidad. Por ejemplo, recientemente permitió conjurar una amenaza al mercado del cobre. Gracias a una buena gestión diplomática, se hizo ver a las autoridades chinas que la concesión de preferencias al cobre procedente de las repúblicas de Asia Central, en perjuicio del cobre chileno, constituía una violación de la cláusula de la nación más favorecida, piedra angular de la normativa de la OMC, que prohíbe este tipo de discriminación, salvo en casos muy calificados. Es evidente que, como nuevo miembro de la OMC, a China le interesa evitar disputas comerciales que puedan llevar a litigios ante dicho organismo. Por otra parte, no descartamos la posibilidad de un TLC entre Chile y China en el futuro. Ello requeriría, a nuestro juicio, que China demuestre que ha hecho progresos sustanciales en el ámbito de las disciplinas de la OMC. Además, sería interesante en esta perspectiva que Chile viera a China como un todo, considerando, por tanto, la negociación de acuerdos preferenciales tanto con la República Popular China como con Taiwán (Taipei chino) y Hong Kong (región administrativa especial de China)

Otro aspecto muy relevante es el nuevo acuerdo de libre comercio entre Hong Kong y China. Este tratado se firmó porque, aunque se trata de un solo Estado, los territorios aduaneros son separados. Un antecedente interesante para quienes deseen gozar de libre acceso a China es que cualquier empresa de Hong Kong que haya operado por un mínimo de 3 años goza de los beneficios del tratado, de manera que un inversionista puede comprar una empresa existente y convertirla en su instrumento para operar libremente en China. Además, las empresas de Hong Kong están obteniendo amplias licencias de distribución en todo el territorio chino, lo que antes del tratado no era posible. Por ejemplo, un exportador puede recurrir a los servicios de una compañía de trading y servicios logísticos como Li $\&$ Fung (que tiene gran experiencia en negocios con clientes como Almacenes París y Jumbo) y por su conducto vender a múltiples clientes finales a los que de otra manera no tendría fácil acceso ${ }^{7}$.

\section{Gracias al acuerdo de libre comercio las empresas de Hong Kong obtienen amplias licencias de distribución en todo el territorio chino.}

\section{CRECIENTE IMPORTANCIA \\ DEL MERCADO COREANO}

Las exportaciones chilenas a Corea cayeron bruscamente debido a la crisis asiática (1997: 987 millones de dólares; 1998: 384 millones). La recuperación posterior ha sido fluctuante, llegando en 2002 a 710 millones de dólares, lo que sitúa a Corea en el tercer lugar de nuestros mercados asiáticos. La vigencia del tratado de libre comercio Chile-Corea

${ }^{7}$ El autor agradece las valiosas informaciones y comentarios de la Gerencia de Estudios de la Compañía Sudamericana de Vapores (CSAV S.A). 
debería dar nuevo impulso a nuestras exportaciones a ese importante mercado, ya que las preferencias arancelarias que obtendremos no las tendrá ningún competidor. Pero el aprovechamiento efectivo de estas ventajas requiere un trabajo empresarial bien planificado, para que los clientes actuales y potenciales en Corea decidan realizar nuevos negocios con proveedores chilenos. Es un desafío muy interesante, que incluye oportunidades para diversificar la canasta de productos e incrementar la proporción de los que incorporan mayor valor agregado.

\section{Para exportar en forma competitiva hay que abrir el comercio en ambas direcciones.}

\section{ASIA-PACíFICO COMO PROVEEDOR}

El análisis del comercio exterior tradicionalmente pone énfasis en las exportaciones y olvida el tema de las importaciones. Esto es consecuencia de la vieja mentalidad mercantilista, para la cual exportar es lo único ventajoso, mientras que siempre que sea posible habría que sustituir las importaciones. Pero, para exportar de manera competitiva es necesario abrir el comercio exterior en ambas direcciones, como se ha hecho en Chile desde mediados de los años setenta. De este modo, los exportadores tienen acceso a los mejores bienes de capital e insumos que se encuentran en diferentes mercados, y los consumidores pueden acceder a mayores niveles de bienestar, y escoger libremente los productos que prefieren. Esto vale, por supuesto, si las reglas son claras y se respetan, no así si se trata de prácticas de depredación del mercado o de subterfugios como la subfacturación.

En el intercambio chileno con AsiaPacífico, las importaciones tienen un papel relevante, aunque en general el comercio exterior con la región genera un superávit que permite financiar en la balanza comercial los flujos deficitarios, que se dan principalmente en el intercambio con el área Mercosur.

Entre los mercados de origen más importantes se da una segmentación de productos, en la que la gama más alta proviene principalmente de Japón, la media de Corea y Taiwán y los países más avanzados del sudeste asiático, y la media-baja y baja de China, Vietnam y otras economías en desarrollo. Esta distribución no es estática, sino dinámica, debido a la fuerte competencia entre los proveedores de distintos países. Corea y Taiwán hoy incursionan en rubros de tecnología avanzada, antes casi exclusivamente japoneses, mientras China trata de dejar atrás su condición de proveedor de manufacturas simplemente baratas y, sin abandonar este segmento, está ingresando a rubros de mayor valor agregado, sea con productos finales, o con partes de estos que se integran a manufacturas de diversos orígenes (por ejemplo, unidades de disco duro para computadores personales).

En la gama más alta de productos, Japón sigue dominando mediante marcas ampliamente conocidas para el público, 
tales como Sony, Honda, Toyota Canon o Yamaha entre los bienes de consumo durables, y de bienes de capital avanzados, por ejemplo, máquinas-herramienta inteligentes (robots) de la industria Fanuc, materiales especiales Kyocera, instrumental médico y de laboratorio, y productos de la industria biotecnológica. En los bienes más complejos, la competencia se da en términos de calidad más que de precio, ya que los competidores son generalmente firmas de otros países que producen bienes de alto costo, como Alemania o Estados Unidos. No obstante, los precios no dejan de tener importancia. Por ejemplo, para mantener la competitividad de las marcas japonesas, algunas industrias usan componentes importados de países que tienen costos inferiores, o trasladan a ellos la producción de los modelos más simples. Por esta razón, un importador chileno de una marca japonesa, por ejemplo en el rubro electrónico, podrá comprobar que el origen de algunos de los modelos de productos podrá ser Malasia, Tailandia o China, aunque haya hecho su pedido a Japón.

Empresas de Corea y Taiwán han entrado agresivamente a disputar terreno a las compañías japonesas, inicialmente sólo con argumentos de precio, pero más recientemente también ofreciendo productos avanzados en diseño y calidad. Las marcas coreanas aparecieron en los mercados internacionales alrededor de los años ochenta y ocuparon el segmento bajo del mercado, por ejemplo, automóviles de diseño básico, como el Pony de Hyundai. Pocos pensaron entonces que actualmente dicha marca alcanzaría un alto presti- gio, junto con Samsung, LG y otras. En el área de insumos industriales y de bienes de capital, la industria coreana es líder en acero (Pohang Steel, POSCO), en construcción naval (astilleros Hyundai y Daewoo) y en chips avanzados (Samsung Electronics y otros). Entre los productos taiwaneses, los PC marca Acer y los semiconductores TSMC, líderes en la industria, constituyen una nueva etapa de competitividad de Taiwán.

\section{Parte importante de las manufacturas chinas son aún productos similares a los de marcas internacionales.}

China inició su dinámico despegue industrial en el segmento bajo de productos, llegando pronto a dominar en rubros como calzado, vestuario y juguetes y bicicletas (o componentes para armar bicicletas de modelos básicos). Las marcas chinas recién comienzan a aparecer en los mercados internacionales, por ejemplo, los PC Legend y los electrodomésticos Haier. Pero se trata todavía de excepciones, ya que en general las industrias chinas trabajan con el sistema OEM (Original Equipment Manufacturer) para terceros, generalmente empresas multinacionales o cadenas comerciales que venden marcas propias. En el esquema OEM, estos clientes contratan manufacturas bajo determinadas especificaciones de diseño y calidad. Otra parte, todavía importante, de las manufacturas chinas consiste en productos similares a marcas internacio- 
nales, sin que necesariamente sean copias exactas o, en algunos casos, lisa y llanamente falsificaciones o reproducciones no autorizadas de productos de marca. Por ejemplo, la filial china de la automotriz Volkswagen descubrió hace poco que una compañía china de la que es socia estaba produciendo cantidades adicionales de componentes de marca para instalarlos en diversos modelos de vehículos chinos que compiten con Volkswagen. De acuerdo con las normas de la OMC, el gobierno chino se ha comprometido a poner fin a este tipo de infracciones a los derechos de propiedad intelectual e industrial. Sin duda, este tema constituye una lucha larga y difícil, pero en la medida en que China logre ascender en la escala de valor de las manufacturas, los incentivos para la falsificación deberían ir mermando gradualmente.

\section{Quienes tienen mayor poder} comprador pueden negociar grandes volúmenes de productos diseñados y fabricados para ellos.

El negocio de importación tiene diversas modalidades, según los países y empresas de origen. Por lo general, las grandes marcas pertenecientes a empresas multinacionales normalmente operan a través de filiales comerciales o de representaciones locales que importan sus productos y los comercializan en el país, lo que obviamente da gran seguridad a mayoristas y minoristas o a compradores industriales. En rubros de consumo masivo, como los que comercializan las grandes tiendas y cadenas de supermercados, los departamentos de adquisiciones suelen enviar compradores a los países de origen, sin perjuicio de usar Internet y las ferias.

Pero quienes tienen mayor poder comprador tienen la opción de negociar grandes volúmenes de productos diseñados y fabricados especialmente para ellos, para lo cual pueden recurrir a los servicios especializados de compañías de trading, como las referidas empresas sogoshosha o a empresas como la ya mencionada Li \& Fung, que ha transformado este negocio desde la simple intermediación de compras de productos standard al trading de servicio total, abarcando todas las etapas de la cadena de abastecimiento, hasta la entrega a satisfacción del cliente. Gracias a este tipo de práctica, a manera de ejemplo, la ropa que se vende en cadenas como Johnson's, Almacenes París u otras, no parece "china" ni norteamericana, sino similar a la hecha en Chile, ya que las especificaciones que cumple el proveedor asiático corresponden a las preferencias del consumidor chileno, definidas por el cliente local.

\section{VÍAS PARA IMPULSAR LAS RELACIONES COMERCIALES Y DE INVERSIÓN}

Para finalizar este análisis, sugerimos un conjunto (no exhaustivo) de políticas públicas y privadas que podrían potenciar la aproximación del sector privado a los mercados comerciales y a las múltiples oportunidades de negocios que ofrece la región Asia Pacífico. 


\section{Promover la imagen económica del país}

Esta es una tarea tanto pública como privada, ya que las empresas en su conjunto constituyen el sistema de mercado que impera en el país, columna vertebral de la economía nacional. Dar a conocer en el exterior la buena gestión y progreso de la economía, que ya estamos acostumbrados a considerar esencial en las tareas de gobierno, es una tarea central de la política de comunicaciones y de la política exterior económica, pero también es una misión de las organizaciones empresariales. Se trata de mostrar el manejo responsable de la economía del país, la solidez de las cuentas externas, la vigencia del Estado de derecho, el respeto de los derechos de propiedad y a los contratos válidamente celebrados, los bajos niveles de conflictividad laboral, las garantías a la inversión extranjera, y el creciente consenso sobre los beneficios de la economía de mercado. Esta tarea debe comprometer la diplomacia presidencial (especialmente en las giras del presidente), a la Cancillería, a los ministerios económicos y a entidades empresariales tales como la Sofofa, la Asociación de Bancos, la Cámara Nacional de Comercio, la Corporación Nacional de Exportadores, y otras.

El resultado debe ser mantener una imagen diferenciada de la visión generalmente negativa que prevalece sobre América Latina en el exterior, aunque Chile no puede negar su identidad latinoamericana y, en definitiva, una mejoría de la región sería favorable para nuestros intereses.

\section{La promoción de la imagen económica del país es tarea tanto pública como privada.}

\section{Mejorar las redes de transporte}

El nivel de eficiencia de las redes de transporte incide directamente en la competitividad de los productos que se comercian con el exterior. En Chile, el tema comienza con el transporte caminero y ferroviario y los niveles de eficiencia de los puertos, que si bien han mejorado, sobre todo en los terminales concesionados al sector privado o de propiedad privada, todavía deben llegar a niveles de mayor productividad.

En el caso de la región Asia-Pacífico, la importancia del transporte de productos a granel es muy grande. Por esta razón, los servicios de transporte marítimo de estos productos, como minerales y productos forestales, se encuentran más desarrollados y las condiciones son en general convenientes.

En el caso de la carga en contenedores, las frecuencias son menores, y en muchos casos se requiere hacer transbordos de carga en puertos como Los Angeles, lo que exige una coordinación exacta de itinerarios para controlar eventuales costos adicionales y, en el caso de las cargas refrigeradas, no romper las cadenas de frío. En Asia, suele requerirse transbordos adicionales en grandes puertos de transferencia, como Hong Kong o Singapur, antes de llegar a los destinos finales.

En ambas áreas, una política dinámi- 
ca nacional y regional de desarrollo de la infraestructura, más allá de lo que ya se ha logrado, permitiría mejorar la competitividad de los productos chilenos $\mathrm{y}$, además, ofrecer servicios de carga a los países vecinos o partes de ellos (provincias del oeste de Argentina, Estados del interior de Brasil).

\section{Para los empresarios es vital acortar los tiempos de viaje $y$ reducir el costo de los traslados a la región Asia-Pacífico.}

En el área del transporte aéreo, la carga se transporta sólo cuando la incidencia del flete en los precios finales así lo permite. Hasta ahora estos flujos son pequeños y se hacen por vías indirectas, por ejemplo, con transbordos en Europa, y para llegar a destinos finales subsisten problemas, como el manejo de cadenas de frío. Para citar un caso, hay muchas dificultades para llegar con salmón fresco al sudeste asiático, motivo por el cual las exportaciones deben limitarse a productos congelados de menor precio, que se transportan por vía marítima.

Para los empresarios es vital acortar los tiempos de viaje y los precios de los pasajes aéreos a la región Asia-Pacífico. Las altas tarifas y tiempos de viaje de 30 y más horas en cada dirección siguen constituyendo factores que disuaden a muchos cuando se trata de hacer negocios con la región, sobre todo en el caso de las PYME, que no pueden pensar en establecer oficinas estables en las gran- des plazas comerciales de Asia-Pacífico. La apertura de la ruta Santiago-AucklandSydney por LAN Chile y Qantas es un gran progreso, pero las frecuencias todavía son insuficientes para las necesidades de los viajeros de negocios. Mientras ésta sea la situación, seguiremos dependiendo de las conexiones vía Estados Unidos (poco convenientes, especialmente después del 11 de septiembre de 2001) o Europa (afectadas por la escasez de vuelos directos entre Santiago y los principales aeropuertos europeos).

\section{Conocer el mundo de los negocios} $y$ disminuir la brecha cultural

Los empresarios chilenos, en general, no dan mucha importancia al análisis académico del mundo de los negocios en diversos mercados externos, ni al conocimiento relativamente sistemático del entorno cultural en que les tocará desenvolverse en sus actividades internacionales. Todavía subsiste cierta percepción de que este tipo de esfuerzos es una "pérdida de tiempo". De este modo, con honrosas y notables excepciones, su "mapa cognitivo" y de actitudes para enfrentar a sus interlocutores suele ser insuficiente. Para superar esta situación, las entidades gremiales de los empresarios, las cámaras de comercio y las universidades, entre otras instituciones, podrían desarrollar actividades conjuntas, lo que redundaría en un fortalecimiento mutuo. 
4. Aprovechar la participación chilena en los foros multilaterales

A partir del año 2000, Chile ha tenido una muy destacada participación en el APEC Business Advisory Council (ABAC), por medio de sus representantes titulares Hernán Somerville, Andrónico Luksic C. y Juan Villarzú, y del alterno Francisco Garcés. No obstante, los empresarios nacionales generalmente han prestado escasa atención al valioso trabajo del ABAC, y su participación en las Cumbres de APEC, en que ABAC es protagonista de las conferencias CEO Summit, ha sido esporádica y claramente insuficiente. Esto constituye una pérdida de oportunidades de vinculación, información e influencia, que debería corregirse con oportunidad de la Cumbre del APEC, que tendrá lugar en Santiago en noviembre de 2004.
5. Aumentar el uso de las tecnologías de la información

Por último, pero no por ello menos importante, los empresarios deberían aprovechar de manera mucho más sistemática las innumerables y crecientes oportunidades para tender puentes a través del Pacífico utilizando las herramientas que brindan la tecnología de la información y las telecomunicaciones. Por ejemplo, en las tareas de inteligencia de mercado y de marketing internacional, estas tecnologías pueden multiplicar las oportunidades de acceso a los mercados, aunque por supuesto sin reemplazar los contactos directos con clientes, proveedores, representantes y otros. En el caso de las PYMES, que son las más afectadas por los altos costos de transacción de los negocios que se realizan por las vías tradicionales, las alternativas virtuales deberían ser especialmente relevantes, convirtiéndose en un gran "factor de nivelación" frente a las empresas grandes. 